\title{
Nurse practitioner's perceptions of the impact of the nurse practitioner-led clinic model on the quality of care of complex patients
}

\author{
Roberta Heale ${ }^{1}$, Susan James ${ }^{2}$, Elizabeth Wenghofer ${ }^{3}$ and Marie-Luce Garceau ${ }^{4}$ \\ ${ }^{1}$ Associate Professor, School of Nursing, Laurentian University, Sudbury, ON, Canada \\ ${ }^{2}$ Associate Professor, Director, School of Midwifery, Laurentian University, Sudbury, ON, Canada \\ ${ }^{3}$ Associate Professor, Director, School of Rural and Northern Health Laurentian University, Sudbury, ON, Canada \\ ${ }^{4}$ Retired Professor, Services Sociale, Laurentian University, Sudbury, ON, Canada
}

\begin{abstract}
Aim: To evaluate the organizational processes that influence the quality of care for patients with multimorbidity at nurse practitioner-led clinics (NPLCs). Background: People are living longer, most with one or more chronic diseases (mulitmorbidity) and primary healthcare for these patients has become increasingly complex. One response was the establishment of new models of primary healthcare. NPLCs are an example of a model developed in Ontario, Canada, which feature nurse practitioners as the primary care providers practicing within an interprofessional team. Evaluation of the extent to which the processes within NPLC model addressed the needs of patients with multimorbidity is warranted. Methods: Eight nurse practitioners were interviewed to determine their perception of the quality of care provided to patients with multimorbidity at NPLCs. Interpretive description guided the analysis and themes were identified. Findings: Three themes arose from the analysis, each of which has an impact on the quality of care. The level of patient vulnerability at the NPLCs was high resulting in the need to address social and financial issues before the care of chronic conditions. Dynamics within the interprofessional team impacted the quality of patient care, including NP recruitment and retention, leaves of absence and turnover in staff at the NPLCs had an effect on interprofessional team functioning and patient care. Finally, coordination of care at the NPLCs, such as length of appointments, determined the extent to which attention was given to individual clinical issues was a factor. Strategies to address social determinants of health and for recruitment and retention of NPs is essential for improved quality of care. Comprehensive orientation to the interprofessional team as well as flexibility in care processes may also have positive effects on the quality of care of patients with complex clinical issues.
\end{abstract}

Key words: nurse practitioner; nurse practitioner-led clinic; patient vulnerability; recruitment and retention; social determinants of health

Received 26 April 2017; revised 30 November 2017; accepted 15 December 2017; first published online 9 January 2018

\section{Introduction}

There is a global trend of people living longer, however, doing so while managing one or more chronic conditions (World Health Organization,

\footnotetext{
Correspondence to: Associate Professor R. Heale, Laurentian University School of Nursing 935 Ramsey Lake Road, Sudbury, ON, Canada P3E2C6. Email: rheale@laurentian.ca
}

(C) Cambridge University Press 2018
2014). Having multiple chronic conditions, or multimorbidity, is complex and challenging for both the patient and their healthcare providers (Bower et al., 2011; Wallace et al., 2015). Traditional family practices have struggled to meet all the healthcare needs of this growing group of patients. In Canada, one response to these challenges is primary healthcare reform, which includes a shift from solo family doctor practices 
to the development of new models of primary healthcare.

Primary healthcare organizational structures and processes have been shown to influence the quality of the healthcare patients receive, as well as health outcomes (Campbell et al., 2009; Kiran et al., 2014). Factors within an organization, such as communication processes and teamwork, as well as community partnerships and patient readiness, all have an impact on patient health outcomes (Russell et al., 2010).

One new model of primary healthcare in Ontario is the nurse practitioner-led clinic (NPLC). NPLCs were established in areas where there were large numbers of people without access to family practices. The model is unique in that nurse practitioners (NPs) are the primary care providers rather than physicians with an NP clinic lead. Patients register at the clinics and are assigned to a primary NP who works with an interprofessional team to provide comprehensive care (Virani, 2012). Additional team members include physicians, who act as primary care consultants and a mix of registered nurses, registered practical nurses, social workers and dieticians (Virani, 2012). The NPs at these NPLCs struggle with the same patient care issues as in other family practice settings, most notably chronic disease management in the care of patients with mulitmorbidity (Virani, 2012).

It is important to evaluate the quality of care in new models of primary healthcare, particularly for patients with the most complex health concerns. Donabedian (2003) developed a framework for the evaluation of quality of care in healthcare systems. When considering organizations, the first two components of this framework are relevant. Structures, the conditions in which care is provided, or the way that healthcare is set up, including material and human resources and organizational characteristics, is the first component. The second is process, which refers to the activities that constitute healthcare such as diagnosis, treatment, patient education and 'clinical efficiency.' An analysis of each component as well as the interplay between them provides insight into the quality of care within an organization.

Given the unique care processes at the NPLCs the quality of care for patients with multimorbidity within the NPLC model should be considered. NPs practicing in the NPLCs can offer insights into the structures and processes specific to the model and their influence on care of patients with multimorbidity. The research question guiding this study was: 'How do nurse practitioners at NPLCs evaluate the impact of the NPLC model on the quality of the care they provide to patients with diabetes and multimorbidity?' An additional goal of this study was to identify potential gaps, or issues in healthcare delivery at NPLCs for further inquiry.

\section{Methods}

This was a qualitative study that used the interpretive description methodology. NPs practicing at NPLCs were interviewed. The purpose of the interviews was to obtain a more in-depth understanding about the impact of organizational characteristics on NP's care for diabetic patients with multimorbidity. A set of questions was developed to help guide the interviews and reflect on the barriers and supports in the care of patients with diabetes and multimorbidity, including the impact of the organizational features of the NPLC model in practice (Table 1).

NPs were recruited to participate in a telephone interview from four NPLCs located in the midnorthern area of the province of Ontario, Canada. The NPLCs were located in urban (two) and rural settings (two), but were chosen for their similarity and in being traditionally underserved areas with high numbers of patients who had no access to primary healthcare services (MOHLTC, 2010). NPs were eligible for interview if they worked either part or full time at one of the NPLCs in the study. The NPs were emailed an invitation to participate in an audio-recorded telephone interview along with a draft of semi-structured questions. They were given the option to be

Table 1 Semi-structured nurse practitioner interview questions

1. What are the barriers in caring for patients with diabetes and multimorbidity?

2. What assists you in caring for patients with diabetes and multimorbidity?

3. How does the NPLC influence the care you provide to diabetic patients with multimorbidity?

4. What would enhance your care of diabetic patients with multimorbidity?

NPLC $=$ nurse practitioner-led clinic. 
interviewed anonymously by a third party should they have felt uncomfortable with the interviewer for any reason, however, none of the NPs chose this option. Eight NPs replied to the email invitation with interest in participation after which they were sent a consent form to sign and a date and time for the interview was set. Interviews were conducted from December 2014 until December 2015. All interviews were conducted by telephone and were $\sim 1 \mathrm{~h}$ in duration. Each interview was audiotaped and transcribed in its entirety by a research assistant and checked for accuracy by the primary researcher (Creswell, 2013). The Laurentian University Research Ethics Board gave ethical approval for the study.

\section{Data analysis}

The interviews were completed and analyzed in the interpretive description tradition (Thorne, 2008). Memos were created during each interview and a summary developed. These documents also served as the basis for reflexivity during the data collection and analysis. Each interview was analyzed as soon as transcripts were prepared. The transcripts were read and re-read, notes were made and important statements were highlighted. Insights from early interviews were incorporated into subsequent interviews. Establishment of an audit trail through the compilation of the research protocol, documents reviewed, correspondence, memos and summaries of interviews enhanced the trustworthiness of the data gathering technique utilized in this study (LoBiondo-Wood et al., 2009).

In keeping with the interpretive description, multiple sources were used to support the final themes (Thorne, 2008). This strategy of triangulation, of cross-checking and verification of themes with other sources, confirmed the credibility of the final themes (LoBiondo-Wood et al., 2009). Participants were given the opportunity to review and provide commentary on preliminary analysis. Two participants responded and no edits were requested.

\section{Findings}

The interviews detailed NP's perceptions of their practices at NPLCs. Themes were developed which offer insight into the impact of the NPLC model in the quality of care of patients with multimorbidity.

\section{Patient vulnerability}

The NPs described how the patient demographics and the resources available to them at the NPLCs influenced the development of a therapeutic relationships and the care that was provided. As the NPLCs were established in areas with a few primary healthcare services it is not surprising that many patients who registered in the NPLCs had not had consistent primary healthcare for years. They often had uncontrolled, or undiagnosed chronic conditions. The NPs identified that this resulted in lack of knowledge and understanding of medical conditions with many patients who became overwhelmed with the complexity of their treatment plans and the relationship of their various conditions.

The NPs identified the vulnerability of the patients at the NPLCs. In healthcare, vulnerability commonly seen as a patient's reduced ability to protect themselves from harm; an obstacle to flourishing (Gjengedal et al., 2013). Those who are vulnerable often have lower levels of education, higher stress, lower income and social exclusion (Braveman and Gottlieb, 2014). Vulnerable patients continue to have difficulties maintaining a healthy lifestyle and have worse health outcomes (Health Quality Ontario, 2012).

In the NPLCs, the NPs found that a higher level of care was required for some conditions than others, which patients found daunting. For example, diabetes care requires medications and lifestyle changes plus ongoing monitoring (Canadian Diabetes Association, 2013) while many other conditions require mostly pharmacological treatment. Patients were sometimes fearful of some of the treatment options, such as insulin injections.

The NPs adapted their practices recognizing the pressures patients are under and working toward optimal therapeutic relationships.

I think the important thing to remember with these people with chronic diseases is to try to get them in here regularly but to be very non-judgmental about the things they don't want to change in their life.

(NP2)

The NPs also identified that many patients lack the financial and social supports required to implement the treatment plans for their conditions. Patients were not always able to afford medication, devices or even appropriate food. 
It is the finances. A lot of the patients that we have here don't necessarily have the resources to pay for their supplies.

Mental health issues, such as depression, were often linked to lack of social supports and were seen as an additional barrier to self-care of multiple chronic conditions. This situation was often exacerbated when patients lived in areas without public transportation and community services and had to rely on family and friends for assistance for things like completing diagnostic tests. Hidden costs of attending community services were also identified.

A lot of them don't have access to transportation just cause we don't have a public bus system, or taxi service in the area so they either rely on family, friends, when they can...It impacts what I can do as a provider or what I can recommend.

And for some it's as simple as they have to pay for parking at the Diabetes Education Centre. And they don't here (the NPLC), so there are barriers to using some of the community resources.

The NPs indicated that urgent social and economic issues took priority over the management of the patient's multiple chronic illnesses, which negatively impacted the quality of care of these conditions.

\section{Interprofessional team functioning}

The NPs identified benefits to the interprofessional team approach within the NPLC model. They value their ability to work autonomously with their own panel of patients within the interprofessional team.

Even just the co-workers, we bounce ideas off of each other. So it is very supportive but at the same time we have, each of us have our own freedom to - we each manage things in different ways, right.

(NP1)

The NPs identified that utilizing the skill mix of the team and giving the opportunity for each team member to work to their full scope of practice can result in fewer community referrals and less disruption of care to patients during times of staff turnover or vacancies. For example, the NPLC dietician may be able to provide counseling about diabetes self-care in lieu of referral to a Diabetic Education Centre, or the social worker may be able to provide counseling to patients.

NPLC is very grateful to have a social worker on hand. Because we're in a rural community I find that there's a lot of undiagnosed mental health. Or even just to learn how to cope with the new diagnosis, it comes very handy.

Definitely having the multidisciplinary team that we have access to. The dietician, pharmacist and even times a social worker is a benefit if we have an older frail individual in the community that needs support etc.

(NP1)

Many patients are not accustomed to working with an interprofessional team, which has created some barriers to engagement of team members other than the primary NP.

Some of them (patients) are very resistant, but for those who we've convinced to try one visit have been continuing. It definitely works and helps.

(NP6)

Although a lot of patients are attached to their primary care NP it's often for the benefit of the patient to see the other professional as well.

And it also depends on what the client wants. So if we have a diabetic, overweight, hypertension patient we would offer to - please see the dietician, see our nurse for blood pressure checks. We do have an RPN here who does blood work. So it all depends on what the client is willing to do. So at the bare minimum they'll usually just see the NP but if they're agreeable, we're definitely hoping they'll see other members.

(NP1)

NPs discussed the level of functioning of the team members and the impact on care. In cases where team members' expertise is utilized to the fullest, 
and they are easily accessible, the NPs describe better care delivery. They described the need for clarity of roles and responsibilities and the need to adapt practice to the specific professional relationships among team members:

We're looking at our end and getting them (RNs, dieticians) to work to full scope... if they can do some of the simple little things. If we have a diabetic, they automatically go to the nurse, ideally. It takes the burden off the NP.

It definitely depends on whichever physician you have working in the clinic. They have their own differences in how they practice.

(NP1)

Even within the NP group, it's necessary to negotiate and understand other's roles.

Yes (will do home visits). But it also depends on each practitioner because there's some that don't really feel that that's part of their role or they might not want to. So that's their decision. Each NP may or may not but our social worker does do it, our RPN and our RN will.

(NP1)

Skill level, confidence in caring for patients with complex clinical issues, knowing the limit of their knowledge and when to refer, or consult are some factors that were identified. The team offered benefit in that NPs were able to tap into the expertise of the other members of the team as they matured in the role of primary caregiver.

Another thing that assists me in caring is my interdisciplinary team so working with different RNs or different scopes of practice, other NPs just bouncing ideas sometimes is wonderful. My consulting physician. Those are all aspects. Sometimes I can only do so much and I just kinda block so I can use my consulting physician to pick her ideas and see if I'm missing something. That definitely helps that we have that availability.

(NP5)

The interprofessional team functioning is further influenced by changes in team members. The NPs indicated that each of the NPLCs in the study had experienced turnover, or leaves of absence since opening, which impacted the team dynamics. There were changes in the physician collaborators at several sites as well as with other members of the healthcare team. When members of the team with specific expertise leave the clinic, gaps in care arise, which can impact quality of care.

Most of the NPs indicated that there had been gaps in the NP complement while they had been at the NPLC either from unfilled positions, attrition and maternity or sick leaves. The turnover of NPs in the NPLCs and/or unfilled positions has led to sudden shifting of the remaining NPs' patient caseload to another NP who is not as familiar with the individual patients and their plan of care. Learning the details about a number of complex patients with multiple chronic conditions is time consuming for the NPs who remain at the clinic. Patient access to care is reduced as the NPs have to include these additional patients to their workload.

Provider turnover, maternity leave, definitely impacts how we provide care because we have to adapt... and we still have to function to provide primary care.

Many of the NPs felt overwhelmed with the complexity of the care of patients with multimorbidity, coupled with the additional demands of unfilled positions and NP turnover.

So that becomes a little bit more complicated for me. Like, what about the medications, and not having a pharmacist here anymore to help do some of those medication reviews.

(NP5)

\section{Coordination of care}

NPLC organizational approaches to patient access to clinic services have an impact on their care. The NPs all indicated that their booking system had some 'same day' appointment times and some had formally implemented scheduling processes to achieve this. Also, the nature of appointments with the NP at the NPLC also impacts patient care. NPs who were able to determine appointment length and frequency of appointments for each patient indicated that they were able to address many concerns per visit and that their patients had better medical control of their conditions. 
I think that's done individually with patients so the patients we see monthly because they need that extra support or we're trying to figure out their insulin. And other people it's every three months, so I'd say it's a bit more individualized.

(NP8)

We have a little bit more luxury in time. We can seek and schedule longer appointments that your routine clinics I say. I don't schedule my patients 15 minutes. I schedule chronic disease management for 30 minutes. I talk to them about the regularity of coming in. Ultimately it's their decision to come in every 3-4 months, but I do recommend that they follow that schedule...it's to check in on their goal setting and attainment.

(NP7)

However, some NPs had limitations on their ability to determine length of appointments, number of patients seen per day and amount of time for administrative duties, which has led to some feeling overwhelmed.

Another thing that would enhance (care of patients with multimorbidity) obviously would be prioritizing my scheduling and my day or just in regards to my admin time, clinical time, all of that need. Every week I look at my weekly schedule and review. To not be afraid to say I'm feeling overwhelmed and giving myself more admin time.

In some NPLCs, patients may be seen by someone other than their primary NP, or switched to a different primary NP when there is staff turnover. Thus, continuity of care can suffer.

... sometimes they (patients) will see different providers if someone's away on vacation and it impacts them because they're not getting the same message. Everyone works differently, and that's ok, but I find that it does create a barrier for them.

(NP6)

Finally, NPs were able to implement innovative strategies for patient engagement, which they see as a benefit of the NPLC model. This included programs such as telephone follow up or group medical visits.
So the NPLC model in terms of chronic disease management, certainly we follow basic guidelines for all conditions, but on top of that we look at innovative ways of assisting patients to meet their goals and stay on target.

(NP7)

\section{Discussion}

This study highlights some of the key issues impacting the care of patients with multimorbidity at NPLCs. Although the NPLC model is unique, the themes arising offer insight into the impact of the organization of the NPLC model on quality of care of patients, larger issues facing primary healthcare across the globe, as well as some key areas for continued research.

Delivery of care in the NPLC model is negatively influenced by the inability of the NPs to adequately address their patient's socioeconomic problems. The most urgent patient concerns may arise from the social determinants of health including employment (finances) and social isolation (Braveman and Gottlieb, 2014), rather than to manage chronic conditions.

NPs have a long history of working with high patient vulnerability. In fact, a key feature of the NP role in Canada has been '...the intake of vulnerable patients that say they are dealing with a chronic condition.' (Donald et al., 2010: 95). Typical characteristics of NP patients were not taken into account in the development of the NPLCs resulting in a lack of recognition of the impact of the social determinants on the ability to deliver quality care. The NPLC budget provided by the Ontario Ministry of Health and Long-Term Care, does not allow any flexibility in the budget lines and does not include community development funding. Without a formal connection to the patients in their living environment, such as a community outreach program, there is no mechanism for the NPs to address the patient's socioeconomic concerns so that they can then focus on their medical conditions.

In addition to a lack of NPLC processes to assist with care of vulnerable patients, the NPs find it difficult to address their medical complexity. NP education focuses on medical diagnoses and appropriate processes for health promotion and disease prevention in primary healthcare. Clinical 
practice guidelines are condition-specific (eg, Diabetes, COPD, etc.) (Wyatt, et al., 2014). There are no guides that reflect socioeconomic realities and their impact on patient care. Patient care in NPLCs is delivered within the structures and confines of 'medical' primary healthcare and clinic budget and policies. The NPs have difficulty adjusting to this role and maintaining wholistic care, addressing the social determinants, in the face of these constraints.

The World Health Organization (2010) has identified this situation globally and defines it as selective primary healthcare, where health policy and resources of a jurisdiction are on targeted interventions rather than focused on broader strategies to address the actual sources of morbidity and mortality, including socioeconomic disadvantage (WHO, 2010). Policy development to allow for better integration of healthcare with social supports is a first step. This will be different for each jurisdiction, however, in Ontario, it could mean something as simple as a community case worker who is embedded in both the ministry of health, as well as the ministry of social services. Within the NPLCs themselves, resources should be allotted to develop programs and partnerships which reach out to community more effectively to develop such things as transportation programs to appointments and accessibility to prescriptions.

Flexible organizational processes from a provider and organizational perspective, may be helpful in enhancing the care of patients with multimorbidity at NPLCs. NPs expressed feeling overwhelmed with the complexity of the care of patients, in particular as they are the primary care providers. This is exacerbated with multimorbidity and the demands of assuming another NP's patient load. Benner (1984) identified that organizational support structures may be a necessary component to the career development of nurses and may also be the same for NPs. A helpful strategy to mitigate being overwhelmed may be the ability of the NP to slow down patient appointments and add administrative time and additional support when the caseload changes are made.

Difficulties in NP recruitment and retention reflects an international health human resource crisis (OECD, 2016). This concern in NPLCs is threaded throughout all of the themes and has an overarching impact on the quality of care of patients with multimorbidity at the NPLCs.
The effectiveness of NP practice is reduced as NPs struggle to establish a therapeutic relationship with, and readjust to the technical aspects of the care of each new complex patient they have added to their roster (Donabedian, 2003). Repercussions of staff turnover are also felt in the level of functioning of the interprofessional team.

There has been very little research into the efficacy of recruitment and retention strategies for NPs, particularly focused on rural settings. However, it's well known through strategies related to physicians that the community itself, including housing, education, and recreational opportunities, plays a large role (Felix et al., 2003). In addition, financial incentives have been routinely implemented to maintain the primary care physician workforce (OECD, 2016). In April 2017, NPs received their first increase in salary in 10 years (Tetley et al., 2016). The impact of this on recruitment and retention of NPs in NPLCs has yet to be determined.

Policies to ensure consistent increases in remuneration of NPs in NPLCs as well as the delivery of other community-based incentives is an essential consideration to maintain a stability in human health resources in the NPLCs. NPs themselves have a role to play. Advocating for health equity for the patients at NPLCs and for fair and equitable remuneration is a necessary element to NP practice in Ontario, which is often this is best achieved through participation in professional organizations such as the Nurse Practitioners' Association of Ontario (Haydt, 2017).

\section{Conclusion}

This study sought to determine how NPs at NPLCs evaluated the impact of the NPLC model on the quality of the care they provide to patients with multimorbidity. NPs identified a number of benefits and challenges in the care of patients with multimorbidity in NPLCs. Certainly a significant benefit is an increased access of patients to comprehensive primary healthcare services (Virani, 2012). That alone is confirmation of the value of the NPLC model. However, patient vulnerability and the NP turnover pose barriers to quality of care for patients with multiple chronic conditions. Evaluation of the complexity of patients in NPLCs, along with environmental scan of the resources available (eg, Lab draws), may be used to ensure that adequate 
funding is available to clinics with large numbers of vulnerable patients.

\section{Acknowledgements}

Roberta Heale received grants from the Canadian Nurses Foundation as well as a Primary Heath Care System Program Research Fellowship to complete this research, in partial completion of her PhD.

\section{References}

Benner, P. 1984: From novice to expert. Excellence and power in clinical nursing practice. Don Mills, ON: Addison-Wesley Publishing Company.

Bower, P., Macdonald, W., Harkness, E., Gask, L., Kendrick, T., Valderas, J.M., Dickens, C., Blakeman, T. and Sibbald, B. 2011: Multimorbidity, service organization and clinical decision making in primary care: a qualitative study. Family Practice 28, 579-87.

Braveman, P. and Gottlieb, L. 2014: The social determinants of health: It's time to consider the causes of the causes. Public Health Reports 129 (Suppl 2). Retrieved November 28, 2017 from http://journals.sagepub.com/doi/pdf/10.1177/003335491 41291S206.

Canadian Diabetes Association Clinical Practice Guidelines Expert Committee 2013: Canadian Diabetes Association 2013 Clinical Practice Guidelines for the Prevention and Management of Diabetes in Canada. Canadian Journal of Diabetes, 37 (Suppl 1), S1-212.

Campbell, S.M., Reeves, D., Kontopantelis, E., Sibbald, B. and Roland, M. 2009: Effects of pay for performance on the quality of primary care in England. New England Journal of Medicine 361, 368-78.

Creswell, J.W. 2013: Research design: qualitative, quantitative and mixed methods approaches, third edition. Los Angeles, CA: Sage.

Donabedian, A. 2003: An introduction to quality assurance in healthcare. Oxford: Oxford University Press.

Donald, F., Martin-Misener, R., Bryant-Lukosius, D., Kilpatrick, K., Kaasalainen, S., Carter, N., Harbman, P., Bourgeault, I. and DiCenso, A. 2010: The primary healthcare nurse practitioner role in Canada. Nursing Leadership $23,88-113$.

Felix, H, Shepaerd, J. and Stewart, M.K. 2003: Recruitment of rural healthcare providers: a regional recruiter strategy. The Journal of Rural Health 19 (Suppl), 340-46.

Gjengedal, E., Ekra, D.M., Hol, H., Kjelsvick, M., Lykkeslet, E., Michaelsen, R. and Oroy, A. 2013: Vulnerability in health care-reflections on encounters in every day practice. Nursing Philosophy 14, 127-38.

Haydt, S. 2017: Politics and professions: interdisciplinary team models and their implications for health equity in Ontario.
International Journal of Health Services, 1-26. http://dx.doi. org/10.1177/0020731417717384.

Health Quality Ontario 2012: Quality monitor, report on Ontario's health system. Retrieved April 12, 2017 from http://www. hqontario.ca/portals/0/documents/pr/qmonitor-full-report-2012en.pdf.

Kiran, T., Victor, C., Kopp, A., Shah, B.R. and Glazier, R.H. 2014: The relationship between primary care models and processes of diabetes care in Ontario. Canadian Journal of Diabetes 38, 172-78.

LoBiondo-Wood, G., Haber, J. and Singh, M. 2009: Rigour in research. In LoBiondo-Wood G. and Haber J., editors Nursing research in Canada. Toronto, Canada: Mosby, pp. 297-319.

Ministry of Health and Long-Term Care 2010: Rural and Northern Healthcare Framework/Plan, 1-85. Retrieved April 12, 2017 from http://health.gov.on.ca/en/public/ programs/ruralnorthern/docs/report_rural_northern_EN. pdf.

OECD 2016: Health workforce policies in OECD countries. Right jobs, rights skills, right places. Retrieved April 12, 2017 from http://www.oecd.org/health/health-systems/Healthworkforce-policies-in-oecd-countries-Policy-brief.pdf.

Russell, G., Dahrouge, S., Tuna, M., Hogg, W., Geneau, R. and Gebremichael, G. 2010: Getting it all done. Organizational factors linked with comprehensive primary care. Family Practice 27, 535-41.

Tetley, A., Heydon, A. and Agnew, T. 2016: Minister Hoskins commits $\$ 85$ million over three years to interprofessional primary care. NP News and Media. Retrieved March 2, 2016 from https://npao.org/minister-hoskinscommits-85-million-over-three-years-to-interprofessionalprimary-care/.

Thorne, S. 2008: Interpretive description. Walnut Creek, CA: Left Coast Press Inc.

Virani, T. 2012: Interprofessional collaborative teams. Canadian Health Services Research Foundation, 1-142. Retrieved April 12, 2017 from http://www.cfhi-fcass.ca/ Libraries/Commissioned_Research_Reports/Virani-Interprofessional-EN.sflb.ashx.

Wallace, E., Salisbury, C., Guthrie, B., Lewis, C., Fahey, T. and Smith, S. 2015: Managing patients with multimorbidity in primary care. British Medical Journal 350, 27-30.

World Health Organization 2010: Action on the social determinants of health: learning from previous experiences. Retrieved April 12, 2017 from http://apps.who.int/iris/ bitstream/10665/44488/1/9789241500876_eng.pdf?ua=1.

World Health Organization 2014: Global status report on noncommunicable diseases. Retrieved November 28, 2017 from http://www.who.int/nmh/publications/ncd-status-report2014/en/.

Wyatt, K.D., Stuart, L., Brito, J.P., Carranza Leon, B., Domecq, J.P., Prutsky, G.J. and Egginton, J.S. 2014: Out of context: clinical practice guidelines and patients with multiple chronic conditions. A systematic review. Medical Care 52, S92-100. 